\title{
Flexible, diamond-based microelectrodes fabricated using the diamond growth side for neural sensing
}

\author{
Bin Fan ${ }^{1}$, Cory A. Rusinek ${ }^{2}$, Cort H. Thompson ${ }^{3}$, Monica Setien³, Yue Guo ${ }^{1}$, Robert Rechenberg ${ }^{2}$, Yan Gong ${ }^{1}$,
} Arthur J. Weber ${ }^{4}$, Michael F. Becker ${ }^{2}$, Erin Purcell ${ }^{1,3}$ and Wen Li (iD ${ }^{1}$

\begin{abstract}
Diamond possesses many favorable properties for biochemical sensors, including biocompatibility, chemical inertness, resistance to biofouling, an extremely wide potential window, and low double-layer capacitance. The hardness of diamond, however, has hindered its applications in neural implants due to the mechanical property mismatch between diamond and soft nervous tissues. Here, we present a flexible, diamond-based microelectrode probe consisting of multichannel boron-doped polycrystalline diamond (BDD) microelectrodes on a soft Parylene C substrate. We developed and optimized a wafer-scale fabrication approach that allows the use of the growth side of the BDD thin film as the sensing surface. Compared to the nucleation surface, the BDD growth side exhibited a rougher morphology, a higher $\mathrm{Sp}^{3}$ content, a wider water potential window, and a lower background current. The dopamine (DA) sensing capability of the BDD growth surface electrodes was validated in a $1.0 \mathrm{mM}$ DA solution, which shows better sensitivity and stability than the BDD nucleation surface electrodes. The results of these comparative studies suggest that using the BDD growth surface for making implantable microelectrodes has significant advantages in terms of the sensitivity, selectivity, and stability of a neural implant. Furthermore, we validated the functionality of the BDD growth side electrodes for neural recordings both in vitro and in vivo. The biocompatibility of the microcrystalline diamond film was also assessed in vitro using rat cortical neuron cultures.
\end{abstract}

\section{Introduction}

Nervous systems consist of large networks of neurons that communicate with each other both electrically and chemically. Rapid, transient communication in neural circuits primarily occurs via electrical impulses (action potentials) along the axon. Neuron-to-neuron communication occurs chemically through the secretion of chemical messengers, known as neurotransmitters that bind to specific receptors at synapses ${ }^{1}$. The transduction of neurotransmitters among the body's glands, organs, and

Correspondence: Wen Li (wenli@msu.edu)

${ }^{1}$ Department of Electrical and Computer Engineering, Michigan State University, East Lansing, MI, USA

${ }^{2}$ Fraunhofer USA Center for Coatings and Diamond Technologies, East Lansing, $\mathrm{MI}$, USA

Full list of author information is available at the end of the article muscles regulates and influences complex brain functions and behaviors such as emotion ${ }^{2}$, pain response ${ }^{3}$, and mental disorders ${ }^{4}$. Numerous studies have shown that dysregulated or imbalanced levels of neurotransmitters in the brain, such as dopamine (DA), are associated with many neurological disorders and health conditions, such as Parkinson's disease ${ }^{5}$, schizophrenia ${ }^{6}$, and epilepsy ${ }^{7}$. The ability to characterize the real-time DA dynamic release and uptake will contribute to a complete understanding of the mechanism behind these diseases and provide effective therapeutics.

Over the past few decades, tremendous efforts have been made to develop miniaturized electrode sensors based on micro/nanofabrication technologies for multimodal neurotransmitter sensing and neurophysiology recording with improved spatiotemporal resolution. For

\section{(c) The Author(s) 2020}

(c) (i) Open Access This article is licensed under a Creative Commons Attribution 4.0 International License, which permits use, sharing, adaptation, distribution and reproduction c. in any medium or format, as long as you give appropriate credit to the original author(s) and the source, provide a link to the Creative Commons license, and indicate if changes were made. The images or other third party material in this article are included in the article's Creative Commons license, unless indicated otherwise in a credit line to the material. If material is not included in the article's Creative Commons license and your intended use is not permitted by statutory regulation or exceeds the permitted use, you will need to obtain permission directly from the copyright holder. To view a copy of this license, visit http://creativecommons.org/licenses/by/4.0/. 
neurophysiology recording, while extremely popular in their use, metal microwires and silicon microelectrodes suffer from signal instability and degradation after chronic implantation due to inflammatory responses and fibrous encapsulation evoked by electrode insertion and the subsequent relative motion ${ }^{8,9}$. Increasing evidence suggests that the dimension and stiffness of implanted electrodes contribute greatly to tissue damage and fibrosis in chronic implantation ${ }^{10}$. As such, polymer-based electrodes have been explored extensively as an alternative to reduce the mechanical mismatch of the devices with the surrounding tissues ${ }^{11}$. However, the fabrication of polymer-based electrodes with small dimensions and good durability is challenging due to physical limitations, non-hermetic packaging, and tradeoffs between size and mechanical strength. While recently reported carbon fiber microelectrodes (CFMEs) have a small cross-sectional footprint $(8-9 \mu \mathrm{m}$ diameter) and a low stiffness that promise to reduce chronic tissue damage and neuron death upon implantation ${ }^{12-14}$, the fabrication of CFME arrays requires a laborious and time-consuming alignment and the assembly of individual fibers onto prepatterned circuit board substrates, which may not be practical for building high-channel-count arrays. For neurotransmitter sensing, the traditional microdialysis technique $^{15-19}$ allows the detection of chemicals at extremely low concentrations but has a limited temporal resolution on the minute scale. Electrochemical approaches using microelectrodes, on the other hand, can detect faster events and therefore are more suitable for real-time DA monitoring ${ }^{20}$. However, detecting DA electrochemically in living organisms is extremely challenging because other chemical species that coexist with DA, such as ascorbic acid (AA), are 100-1000 times more concentrated than $\mathrm{DA}^{20}$. In particular, $\mathrm{AA}$ has been found to have an oxidation potential very close to that of DA, resulting in a significant interference ${ }^{21}$.

Ideally, the simultaneous probing of neurophysiology and neurotransmitter signals requires a sophisticated, implantable device that is highly sensitive and selective, miniaturized, fast, biocompatible, and long-term stable ${ }^{22}$. At present, most of the implantable sensors are constructed in an electrode configuration, mainly based on carbon materials, including glassy carbon $^{23}$, carbon nanotubes $^{24-27}$, carbon fibers ${ }^{28}$, and micro/nanocrystalline diamonds ${ }^{29-32}$. Among different carbon materials, boron-doped polycrystalline diamond (BDD) has stood out and has found widespread use in neurotransmitter detection, especially for $\mathrm{DA}^{21,33-35}$. Diamond is inherently an insulator, but when doped with boron, the material possesses semimetal electronic properties, making it useful for electrochemical measurements. During film growth, the boron impurity atoms substitute for some of the carbon atoms, resulting in a rough, polycrystalline morphology with grain boundaries at the film surface and a small-volume fraction of non-diamond carbon $\left(s p^{2}\right)$ impurity. Consequently, the electrical conductivity and electrochemical properties of the film surface and the bulk are influenced by the boron doping level, the grain boundaries, and the impurities ${ }^{36}$. Common BDD properties favorable to electrochemical sensing include a wide potential window, a low double-layer capacitance, excellent selectivity and sensitivity without conventional pretreatment, weak molecular adsorption, biocompatibility, and chemical inertness ${ }^{37,38}$. Despite the many benefits of BDD, the mechanical property mismatch between BDD (Young's module of $\left.\sim 10^{3} \mathrm{GPa}\right)^{39}$ and soft tissues $\left(\sim 10^{3}-10^{5} \mathrm{~Pa}\right)^{40}$ is a major obstacle that impedes the development of BDD into fully implantable electrochemical devices. Many efforts have been devoted to developing various fabrication methods to make diamond-polymer structures with reduced stiffness while maintaining the biocompatibility and inertness of the overall devices. For example, Hess et al $^{40}$ reported a method of transferring small BDD patterns, such as contact pads and electrodes, from a silicon substrate onto a polynorbornene-based polymer (PNB) film by removing a $\mathrm{SiO}_{2}$ sacrificial layer between the BDD patterns and the silicon substrate. Later, Bergonzo et al. ${ }^{41}$ used a similar fabrication process to transfer a diamond microelectrode array onto a flexible polyimide substrate for in vivo retinal prostheses. Previously, we also reported a method for patterning and transferring large-scale BDD structures from solid substrates onto Parylene $C$ thin films. The transferred flexible BDD electrodes have been proven effective for detecting various concentrations of DA with a limit of detection (LOD) of $0.5 \mu \mathrm{M}^{42}$.

However, the above transfer methods allow exposing only the BDD nucleation surface as the sensing sites, keeping the growth surface encapsulated by the polymer film. The nucleation surface is the interface between the BDD film and the substrate where diamond deposition is started. The nucleation surface has small diamond grain sizes and a high density of grain boundaries with a large number of impurities, such as $s p^{2}$ carbon, within the boundaries. These non-diamond impurities can significantly affect the electrochemical properties of BDD electrodes. As the film thickness increases, the diamond grain size increases, resulting in more $s p^{3}$ carbon atoms and less $s p^{2}$ impurities. Qi et al. ${ }^{43}$ reported the effects of the BDD film thickness on the electrochemical properties associated with an $s p^{2}$ impurity, including the electron transfer kinetics, the faradaic current of DA, and the oxidation potential separation between DA and AA. This study has shown that 5.5- $\mu$ m-thick BDD has much less $s p^{2}$ impurities than $0.5-\mu \mathrm{m}$-thick BDD, leading to a significantly faster electron transfer process in the $\left[\mathrm{Fe}_{3}(\mathrm{CN})_{6}\right]^{3-/ 4-}$ redox system, a higher faradaic current of 
DA, and a differentiable oxidation peak between DA and AA in differential pulse voltammetry. Therefore, the growth surface where diamond deposition is ended is believed to be more advantageous than the nucleation side in terms of the sensitivity, selectivity, and stability of electrochemical sensing due to the high fraction of $s p^{3}$ carbon.

In this paper, we present a newly developed fabrication method by which the growth surface of a BDD film can be opened up as the electrode site for chemical sensing and neural recording. The use of the exposed BDD growth side as an effective sensing surface differentiates this work from existing methods ${ }^{19-21}$. To demonstrate the significantly improved electrochemical properties of the growth side, we conducted a comparative study where BDD electrode probes were fabricated from either the nucleation side or the growth side of the BDD thin films produced from the same deposition batch, and their properties were compared with each other. We showed that the electrodes made from the BDD growth surface have a rougher surface, a higher $s p^{3}$ fraction, a wider water potential window, and faster dynamic kinetics than the devices made from the nucleation surface. Furthermore, we validated that compared to the nucleation surface, the BDD growth surface shows better sensitivity and a lower surface absorption to DA. Finally, we assessed the biocompatibility of microcrystalline BDD films in vitro and evaluated the recording capability of the flexible BDD growth surface electrodes both in vitro using a cortical neuron culture and in vivo using the primary visual cortex (V1) of a rat.

\section{Results and discussion Fabricated device}

Figure 1a shows the design of the proof-of-concept BDD neural probe in a three-electrode configuration. The device had two channels on a single probe shank, each of which consisted of a circular working electrode (WE) surrounded by a ring-shaped counter electrode (CE). A square reference electrode (RE) was shared by two working and counter electrode pairs. The effective areas of the exposed BDD electrodes were approximately $0.0079,0.028$, and $0.035 \mathrm{~mm}^{2}$ for the WE, CE, and RE, respectively. The scanning electron microscope (SEM) image in Fig. 1c shows a fabricated probe where the growth surface of the BDD film was exposed on the electrode sites. Figure 1d shows a close-up view of the WE and $\mathrm{CE}$. The probe was flexible when using Parylene $\mathrm{C}$ as a substrate and encapsulation material, as shown in Fig. $1 b$.

A flexible BDD probe with the nucleation side exposed was fabricated with the same geometry and compared with the BDD growth side. Corresponding SEM images and Raman spectra (532 nm Laser; HORIBA Scientific Ltd., Kyoto, Japan) of both the nucleation and growth surfaces are shown in Fig. 2a-c. The SEM images show that the growth side of the microcrystalline BDD film has a rougher surface morphology with an average grain size of $0.5 \mu \mathrm{m}$, while the nucleation has a relatively smooth morphology. The Raman spectra show the expected profile of the highly boron-doped diamond films in which the characteristic diamond $\left(s p^{3}\right)$ peaks at $\sim 1332 \mathrm{~cm}^{-1}$ of both devices are broadened and downshifted to $\sim 1305 \mathrm{~cm}^{-1}$. Additionally, two boron doping-related peaks appeared at $\sim 473$ and $1209 \mathrm{~cm}^{-1}$, which are the typical features of heavily doped BDD thin films with doping concentrations on the order of $10^{20}$ atoms $/ \mathrm{cm}^{3}{ }^{44}$. This resulted in highly conductive BDD films that had a bulk resistivity in the range of $5 \times 10^{-3} \Omega \cdot \mathrm{cm}$. The intensity of the boron doping-induced peak on the BDD growth side was higher than that on the BDD nucleation side since the boron dopants are distributed uniformly within the diamond grains and more boron atoms will be doped into a larger size of the diamond grains on the BDD growth side ${ }^{43}$. Unlike the BDD growth side, there was a small peak at $\sim 1470 \mathrm{~cm}^{-1}$ for the BDD nucleation side,

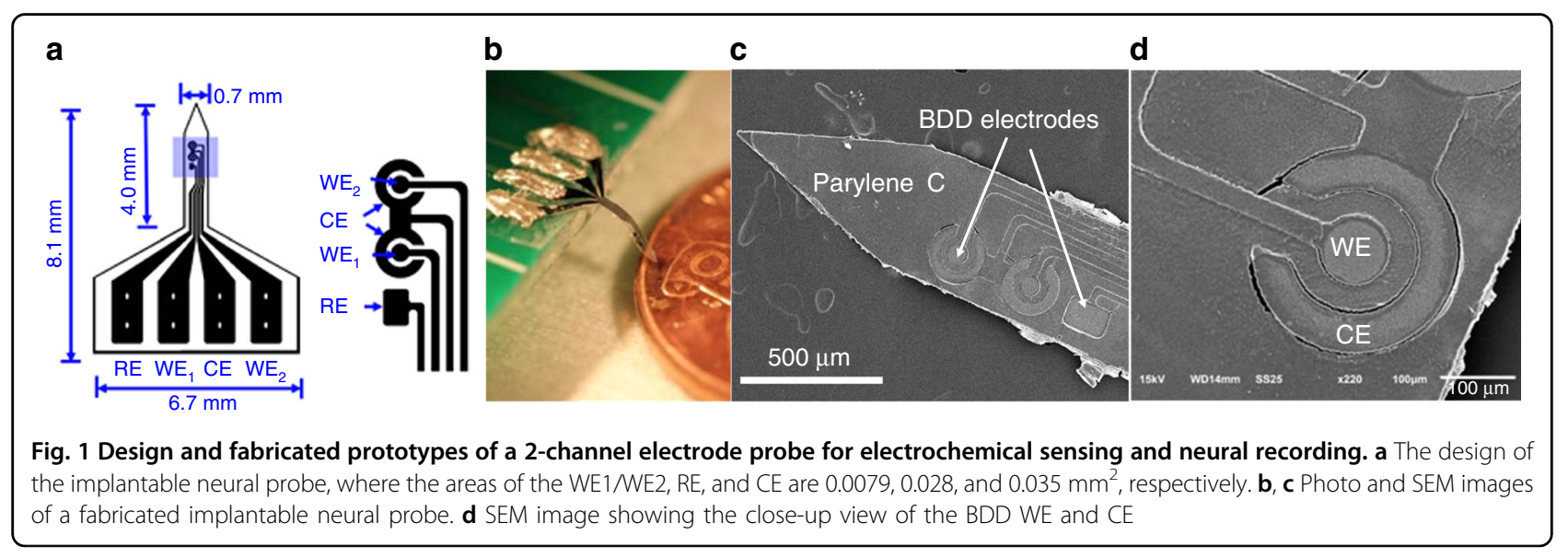



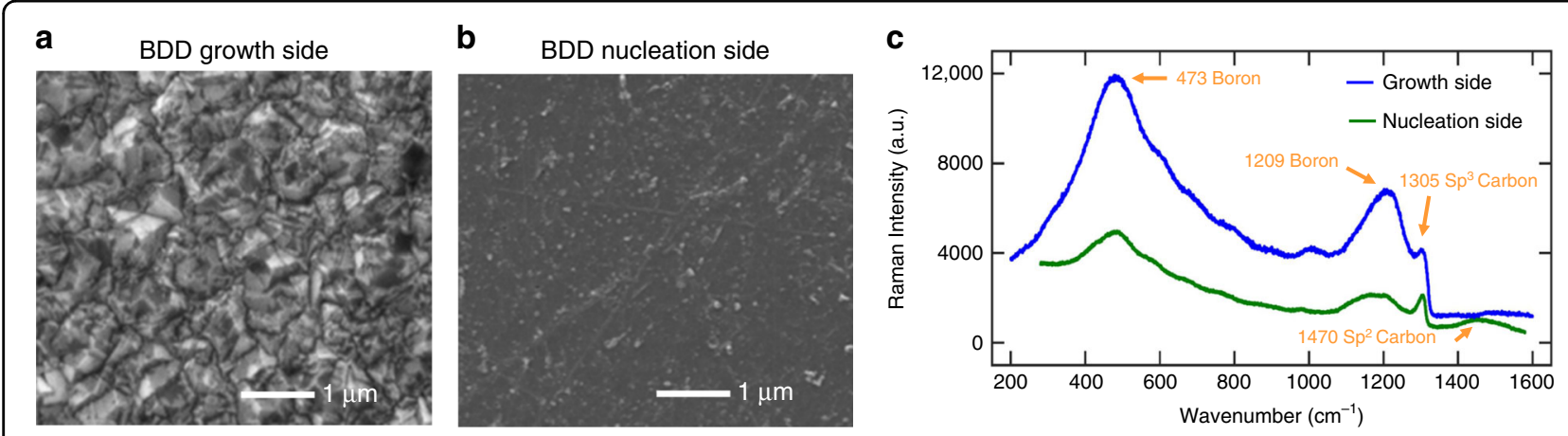

Fig. 2 Material properties of the BDD growth side vs. the nucleation side. SEM images show the surface morphology of a the BDD growth side and $\mathbf{b}$ the nucleation side. $\mathbf{c}$ Raman spectrum of the BDD growth side (blue) and the nucleation side (green)

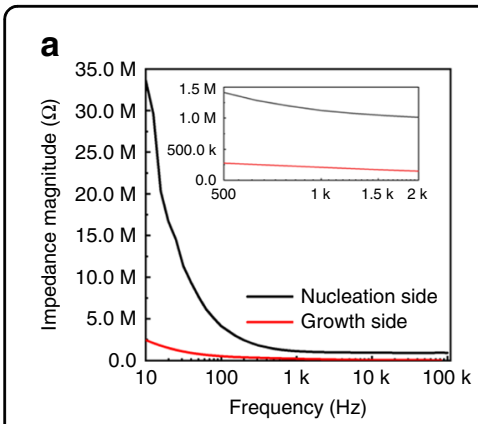

b
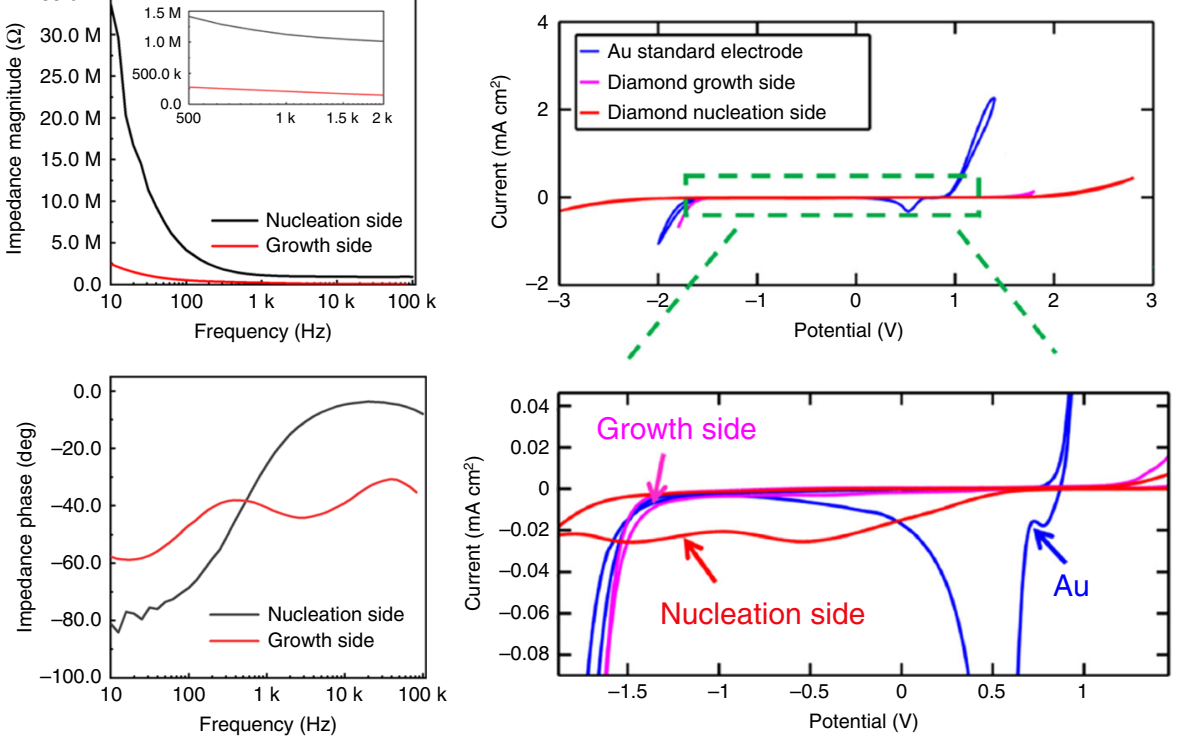
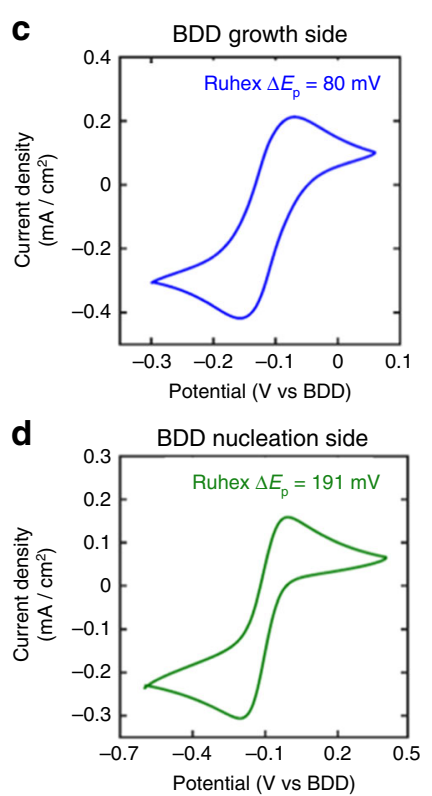

Fig. 3 Electrochemical properties of the BDD growth surface vs. the nucleation surface. a Bode plots showing broadband impedances (10 Hz to $100 \mathrm{kHz}$ ) of the BDD growth side and nucleation side electrodes, measured in a pH 7.4 PBS buffer solution. b Cyclic voltammograms (CVs) of the $\mathrm{Au}$ and BDD growth side and nucleation side electrodes in a $1 \mathrm{M} \mathrm{KCl}$ solution (WE: BDD or Au, CE: Pt, RE: Ag/AgCl). The scan rate is $0.1 \mathrm{~V} / \mathrm{s}$. Voltammograms of the BDD growth side (c) and nucleation side (d) electrodes in the mixture of $1.5 \mathrm{mM} \mathrm{Ru}\left(\mathrm{NH}_{3}\right)_{6}{ }^{2+/ 3+}$ and $1.0 \mathrm{M} \mathrm{KCl}$, scanned at a constant rate of $0.1 \mathrm{~V} / \mathrm{s}$. In this case, both the $\mathrm{CE}$ and RE are made of BDD

indicating a higher concentration of $s p^{2}$ carbon and/or amorphous defects due to the smaller grain size and the larger amounts of grain boundaries. Since these nondiamond impurities are distributed along the grain boundary, the Raman intensity at $1470 \mathrm{~cm}^{-1}$ of the BDD nucleation side was much higher than that of the BDD growth side.

\section{Electrochemical properties}

The electrochemical impedance of the BDD electrodes was measured using electrochemical impedance spectroscopy (EIS) in $0.1 \mathrm{M}, \mathrm{pH}=7.4$ phosphate-buffered saline (PBS) solution at room temperature. Figure 3a shows the broad impedances of the BDD electrodes with the growth side or nucleation side as the sensing surface. The $1 \mathrm{kHz}$ impedance of the BDD growth side electrode is $\sim 207.9 \mathrm{k} \Omega$, which is $4-5$ times lower than that of the nucleation side electrode $(\sim 1123.8 \mathrm{k} \Omega)$. Without changing the size of the electrodes, the BDD growth side has a rougher surface and larger grain size, which can dramatically reduce the impedance, thereby reducing the impedance noise at the electrode-electrolyte interface for neural recording. A comparative study of the potential window and background current among the diamond nucleation side, the diamond growth side, and a commercially available gold electrode was performed using 


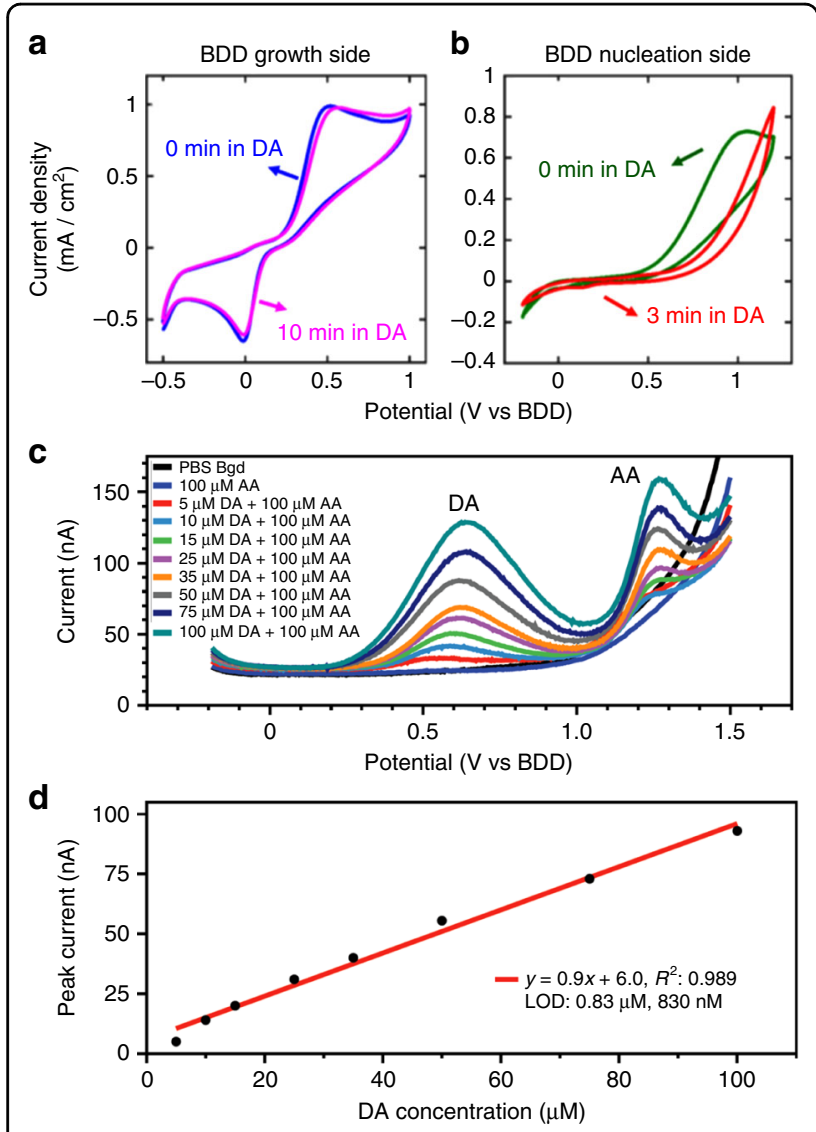

Fig. 4 Validation of the DA sensing capability of the flexible BDD electrode. CVs of the BDD growth side (a) and nucleation side (b) electrodes in the $1.0 \mathrm{mM}$ DA solution at a scan rate of $1.0 \mathrm{~V} / \mathrm{s}$. The voltammograms of both devices after extended soaking in the DA solution were also plotted as a surface fouling test. c SWS of DA detection in the presence of $100 \mu \mathrm{M}$ AA. Measurements were performed using a BDD growth side electrode. $\mathbf{d}$ Linear fitting of the peak current of SWV under various DA concentrations of 5-100 $\mu \mathrm{M}$

cyclic voltammetry $(\mathrm{CV})$ in $1.0 \mathrm{M}$ potassium chloride $(\mathrm{KCl})$ solution with a platinum $(\mathrm{Pt})$ electrode as the $\mathrm{CE}$ and a silver/silver chloride $(\mathrm{Ag} / \mathrm{AgCl})$ electrode as the $\mathrm{RE}$ at a scan rate of $0.1 \mathrm{~V} / \mathrm{s}$. As shown in Fig. $3 \mathrm{~b}$, the BDD electrodes exhibited a featureless background current and wider water potential windows compared to the standard gold electrode. This characteristic permits the detection of chemical analytes in an expanded potential range of operation with reduced interference from the nonfaradaic background current of the electrolyte. The background peaks presented in the nucleation side $\mathrm{CV}$ might be attributed to the reduction in functional groups at the $s p^{2}$ sites $^{45}$.

The effect of the electrochemical kinetics was studied using the one-electron transfer, outer-sphere electron, $\mathrm{Ru}$ $\left(\mathrm{NH}_{3}\right)_{6}{ }^{2+/ 3+}$ redox couple. Figure 3c, d shows the cyclic voltammograms (CVs) of the BDD growth side and nucleation side electrodes, respectively, obtained in a mixture of $1.5 \mathrm{mM} \mathrm{Ru}\left(\mathrm{NH}_{3}\right)_{6}{ }^{2+/ 3+}$ and $1.0 \mathrm{M} \mathrm{KCl}$ at a scan rate of $0.1 \mathrm{~V} / \mathrm{s}$. The separation between the anodic and cathodic peak $\Delta E_{\mathrm{p}}$ has been defined to determine the electrochemical reversibility for a given redox couple. For the one-electron transfer redox couple at room temperature, $\Delta E_{\mathrm{p}}=58.5 \mathrm{mV}$, which is the criterion for a complete thermodynamically reversible process ${ }^{46}$. The $\Delta E_{\mathrm{p}}$ of the BDD growth side was $80 \mathrm{mV}$, which is close to the theoretical value of $58.5 \mathrm{mV}$, thereby indicating the quasireversibility of the reaction. The $\Delta E_{\mathrm{p}}$ of $\mathrm{BDD}$ nucleation was $191 \mathrm{mV}$, indicating that the BDD nucleation side has much slower thermal dynamic kinetics than the BDD growth side. Background CV measurements were repeated at various scan rates of 0.1, 0.5, 1.0, 2.0, and $3.0 \mathrm{~V} / \mathrm{s}$ in $1.0 \mathrm{M} \mathrm{KCl}$ (Fig. S1(a)) to assess the double-layer capacitance $\left(C_{\mathrm{dl}}\right)$ of the BDD electrodes. The average current of the forward and reverse sweeps extrapolated from the CVs at different scan rates was used to derive a linear regression curve (Fig. S1(b)), where the slope defines the double-layer capacitance ${ }^{42}$. Using this method, the double-layer capacitance of the BDD growth side was approximately $10 \mu \mathrm{F} / \mathrm{cm}^{2}$, significantly lower than the $C_{\mathrm{dl}}$ of the nucleation side reported in our previous work ${ }^{42}$, which was $\sim 24 \mu \mathrm{F} / \mathrm{cm}^{2}$. This low capacitance may result from the existence of a space charge region within the near surface of diamond electrodes ${ }^{47}$. The low doublelayer capacitance exhibited by the BDD electrodes permits a reduced charging current and low electrochemical background noise, which has been proven to facilitate chemical sensing of electroactive species (i.e., DA) with sensitivity at least one order of magnitude greater than that of glassy carbon electrodes ${ }^{48}$.

\section{DA sensing capability}

The capability of sensing the inner sphere and twoelectron transfer DA/DAquinone redox couple was compared between the two surfaces. The voltammograms of the BDD growth side and the nucleation side in $1.0 \mathrm{mM}$ DA diluted with a $0.1 \mathrm{M}, \mathrm{pH}=7.4$, phosphate-buffered saline (PBS) buffer solution at a scan rate of $1.0 \mathrm{~V} / \mathrm{s}$ are shown in Fig. 4a, b, respectively. The voltammogram of the BDD growth side electrode (Fig. 4a) shows welldefined, nearly symmetrical, sharp oxidation and reduction peaks. The BDD nucleation side device (Fig. 4b), on the other hand, shows a broad oxidation peak and no significant reduction peak in the voltammogram. This result indicates that the nucleation side has a sluggish electron transfer process due to the non-diamond $s p^{2}$ carbon. A preliminary fouling test was conducted, where both devices were soaked in a $1.0 \mathrm{mM}$ DA solution to study the chemical absorption of different BDD surfaces. The DA concentration used in the experiment is much higher than the expected in vivo concentration in the brain environment ${ }^{49}$ to accelerate the fouling effect. Due 

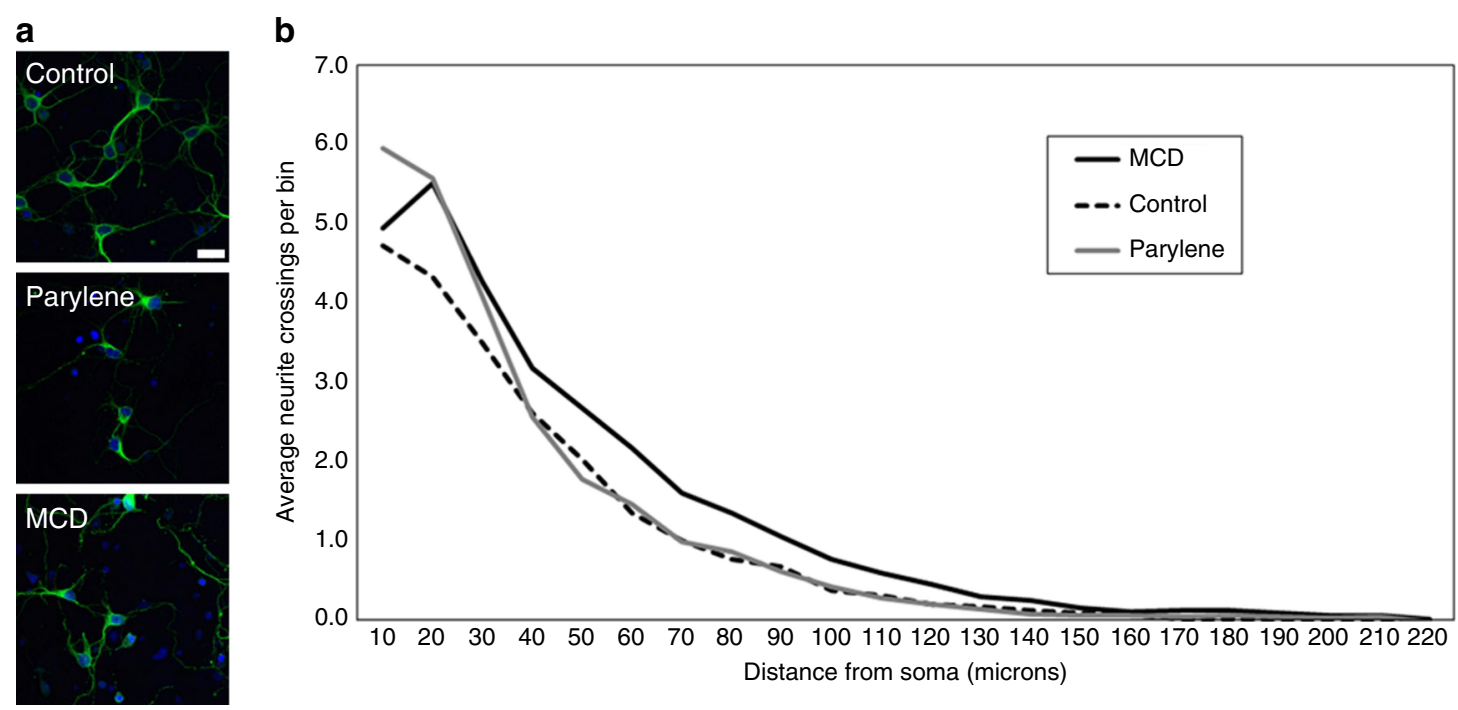

Fig. 5 Morphological response to the electrode materials. a Rat cortical neurons plated on the Parylene C and MCD substrates extended neurites and displayed morphologies that appeared similar to cells plated on control substrates (TUJ1 in green, Hoechst nuclear counterstain in blue). b Quantification of the morphological effects via Sholl analysis illustrates similar responses on all substrates, with a slight increase in neurite extension over longer distances ( $>40$ microns) registered by MCD substrates ( $n=\sim 60$ neurons/substrate). Scale in $(\mathbf{a})=20$ microns

to the non-diamond $s p^{2}$ carbon impurity, the surface of the BDD nucleation side device was fouled within 3 min of DA soaking, resulting in the disappearance of the oxidation peak in the voltammogram, as shown in Fig. 4b. The BDD growth side device shows similar voltammograms before and after the 10-min soaking test, suggesting no significant DA absorption or fouling.

Having validated their better DA sensing performance, we evaluated the selectivity of the BDD growth side electrodes for DA detection in the presence of AA, a major biological interference for in vivo dopamine sensing $^{31}$. In this experiment, DA at various concentrations from 5 to $100 \mu \mathrm{M}$ was serially diluted in a mixture of $100 \mu \mathrm{M}$ ascorbic acid and 0.1 M PBS, and these solutions were used for the square-wave voltammetry (SWV) measurements. The clear peak separation of DA from AA and from each other at different concentrations was observed in the square-wave voltammograms (SWVs) in Fig. 4c, demonstrating the selectivity of the pristine BDD growth side for DA sensing. Furthermore, the BDD growth side electrode exhibits a highly linear response with the correlation coefficient $R^{2}=0.989$ over the tested DA concentration range, as shown in Fig. 4d. A limit of detection (LOD) of $830 \mathrm{nM}$ was estimated based on linear fitting.

\section{In vitro assessment of diamond biocompatibility}

Neurons seeded on microcrystalline diamond (MCD) and Parylene $\mathrm{C}$ appeared qualitatively similar to the control substrate in terms of the adhesion, health, and neurite outgrowth (Fig. 5a). To assess the more nuanced effects on the morphology, a Sholl analysis was performed on $\sim 60$ cells per substrate. The results revealed that the number of neurites that crossed the boundary of the concentric circles (positioned at incrementally increasing $10 \mu \mathrm{m}$ distances from the center of each soma) was significantly higher on MCD than on Parylene $C$ and the control substrates (Fig. 5b, linear mixed models test: $p<$ 0.05). Notably, the first 20 microns displayed a slight upward trend in neurite crossings that was not evident under the other conditions tested; this may be explained by the biological variability. The "maximal distance" measurement likewise indicated that neurons plated on MCD extended longer neurites than cells cultured on the control and Parylene substrates (data not shown; linear mixed models test: $p<0.05)$. The average "number of branching" was statistically higher for Parylene and MCD than for the control, where MCD facilitated the most robust arborization (data not shown; linear mixed models test: $p<0.001)$. Last, the average sum of crossings and the average sum of branchings showed no statistical significance between the substrates (data not shown; linear mixed models test: $p>0.05$ ). Additionally, positive caspase- 3 staining was not detected in any of the substrates tested, indicating a similar support of the viability (the antibody was validated in preliminary tests, data not shown). While the underlying mechanism has yet to be determined, it is possible that the topographical cues presented by the granular MCD surface may promote neuronal maturation and neurite elongation ${ }^{50,51}$. However, the underlying mechanism has yet to be determined in future studies, and it is possible that the surface 
a

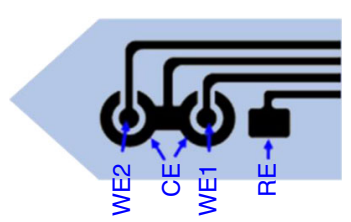

b

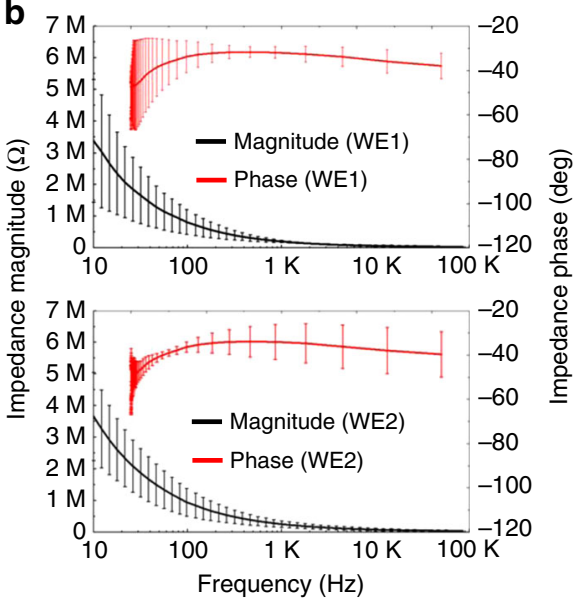

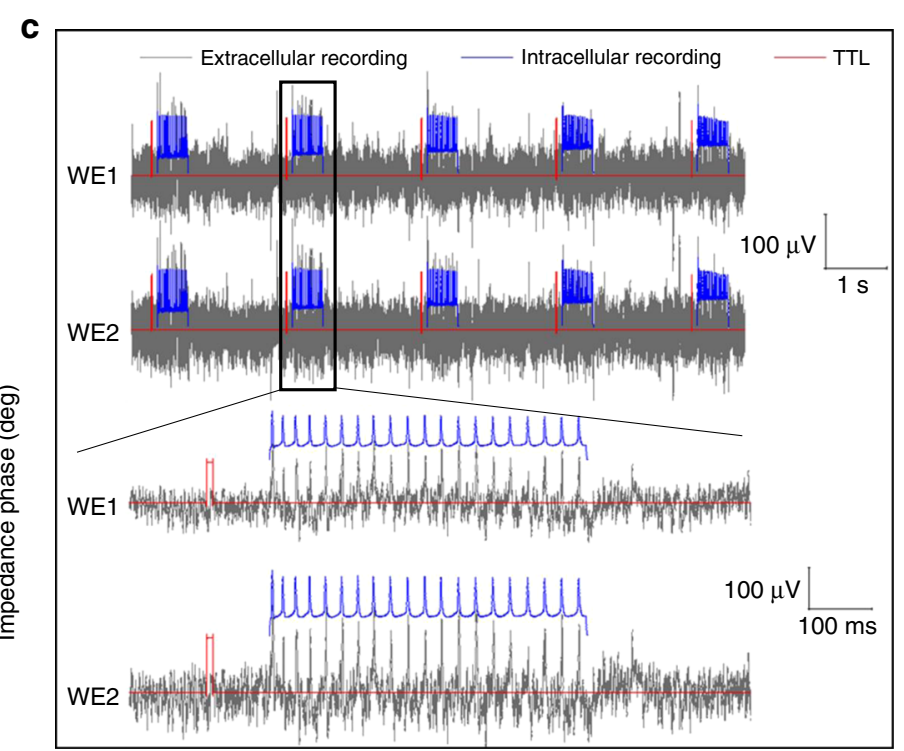

d

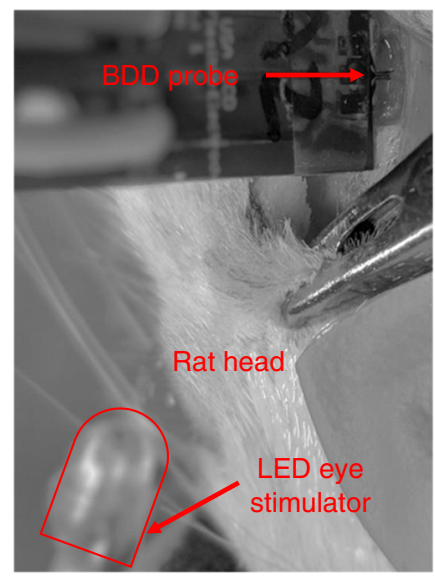

e

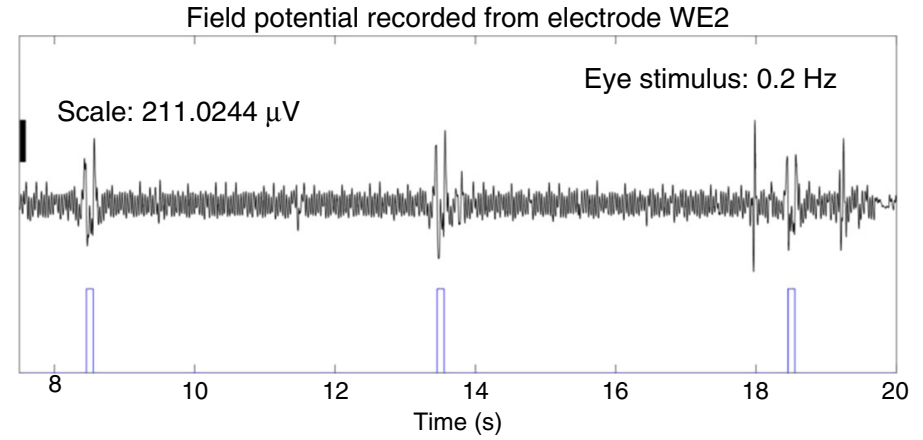

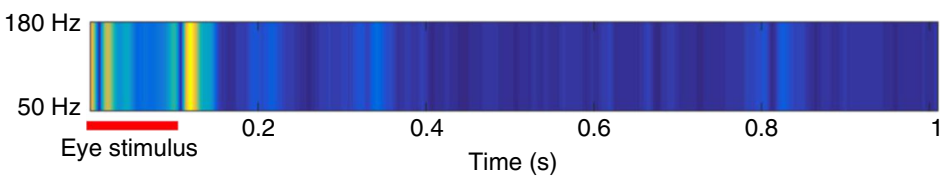

Fig. 6 In vitro and in vivo extracellular recordings using flexible BDD growth surface electrodes. a Electrode layout. $\mathbf{b}$ Bode plots showing impedances $(10 \mathrm{~Hz}$ to $100 \mathrm{kHz})$ of the WE1 and WE2 BDD electrodes measured in the pH 7.4 PBS buffer solution (sample size: $n=4)$ ). c Extracellular recording from the cultured cortical neurons, showing that the spikes detected by the BDD electrode are time-locked to the intracellular activity registered by the patch electrode. $\mathbf{d}$ Setup of in vivo neural recording in the $\mathrm{V} 1$ of a male rat. e Visually induced field potential recorded through the BDD electrode WE2 (top). The color chart (bottom) shows the spectrogram $(50-180 \mathrm{~Hz})$ of the recording within the 1-s cycle. The square wave is the pulses of the eye stimulus

chemistry, material stiffness, and/or conductivity also play a role in this result ${ }^{52,53}$. Overall, the MCD and Parylene C substrates performed as expected in vitro and supported neuronal growth and maturation similarly to the control conditions.

\section{In vitro extracellular recording}

The electrochemical impedance of the WEs was measured using EIS in a room temperature PBS solution $(0.1 \mathrm{M}, \mathrm{pH}=7.4)$. Figure $6 \mathrm{~b}$ shows that the average $1 \mathrm{kHz}$ impedance $(n=4)$ of the WE1 and WE2 is approximately
254.2 and $203.4 \mathrm{k} \Omega$, respectively, which are suitable for neurophysiology recording. The intracellular spiking activity of cultured neurons detected with patch-clamp electrophysiology was highly coincident with the extracellular activity recorded from the two-channel flexible BDD electrode probes (Fig. 6c), demonstrating that the flexible electrodes are capable of recording the extracellular activity of proximal neurons. Previous studies have reported simultaneous intra/extracellular "groundtruth" recordings using comparable configurations, such as glass pipette electrodes inserted near implanted 


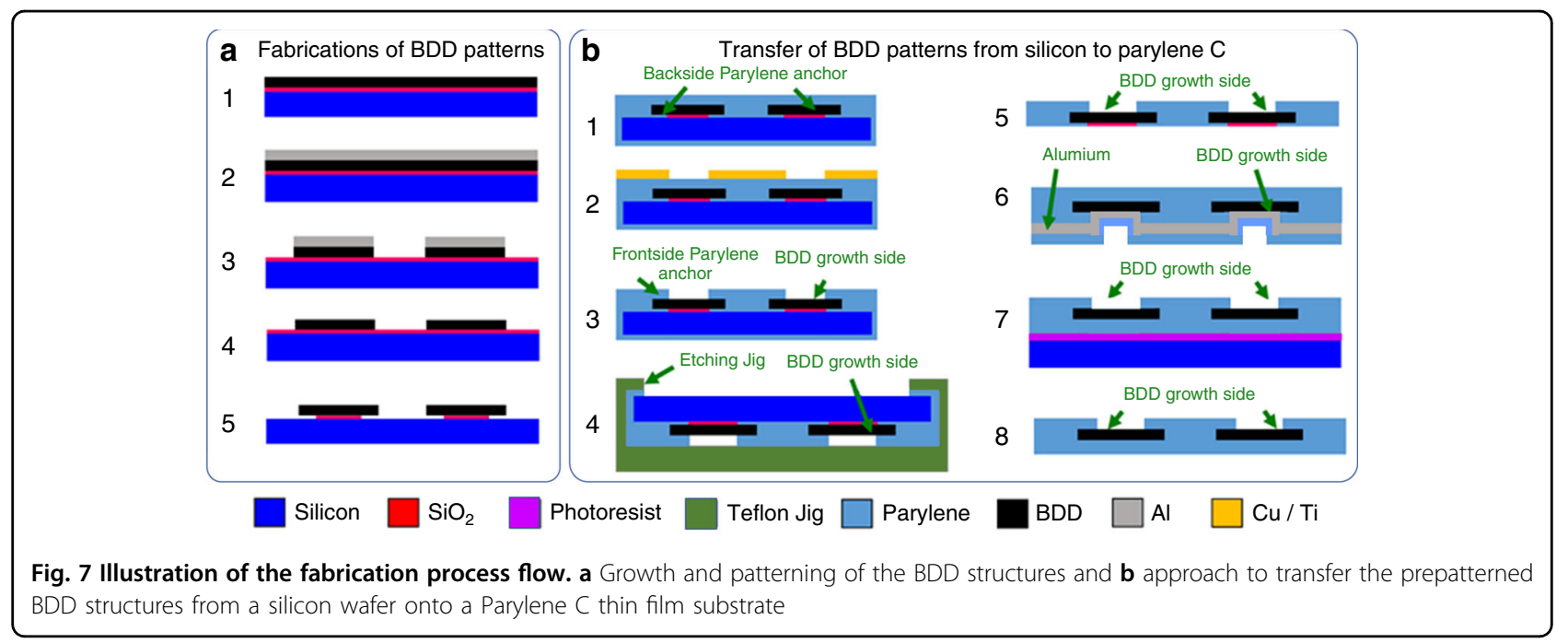

tetrodes in vivo ${ }^{54,55}$ or through the use of microelectrode arrays as neuronal culture substrates in vitro ${ }^{56,57}$. Based on visual estimates of the peak-to-peak noise floor, the signal-to-noise ratio (SNR) of the flexible BDD devices appeared somewhat lower (SNR 2) than traces reported in alternative setups (SNR $\sim 3-4$ in ref. ${ }^{54}$ ). While the purpose of the in vitro recording was to provide proof-ofprinciple for subsequent in vivo use, the SNR of ParyleneBDD electrodes may be further improved in future studies by reducing external noise or optimizing device design (e.g., increasing the surface area of recording sites to reduce impedance and thermal noise).

\section{In vivo electrophysiology recording}

Figure $6 \mathrm{~d}$ shows the setup of in vivo experiments using the rat model. The probe was inserted in the left lobe of the $\mathrm{V} 1$ region at a penetration depth of approximately $1 \mathrm{~mm}$. During the experiments, the right eye of the rat was stimulated optically using a blue LED at a $100 \mathrm{~ms}$ pulse width and a $0.2 \mathrm{~Hz}$ frequency. The BDD electrode that is the closest to the probe tip (WE2) was used to record spontaneous neural responses from the left V1. Figure 6e shows the visually induced local field potential recorded through WE2, where elevated field potentials were detected shortly after visual stimulation, demonstrating stimulus-induced neural activity. The recorded field potential signal was truncated to a 1-s trial (total of 240 trials), averaged across 240 trials, and mapped onto a color-coded time-varying energy distribution (spectrogram) in the gamma band $(50-180 \mathrm{~Hz})$ within the 1 -s time window. As shown in Fig. 6e (bottom), higher gamma band energy was observed within a $50 \mathrm{~ms}$ window post-stimulation, which is consistent with our previous observation $^{58}$. While preliminary, this result suggests that the BDD electrodes are capable of recording neural activity from living tissues.

\section{Methods and materials \\ Device fabrication}

Figure $7 \mathrm{a}$ illustrates the fabrication process flow for BDD synthesis and patterning. Four-inch-diameter silicon wafers were cleaned followed by chemical vapor deposition of $1 \mu \mathrm{m}$ silicon dioxide. Then, the wafers were scratch seeded, and microcrystalline BDD films were grown on the wafers using a $915 \mathrm{MHz}$ microwave plasma-assisted chemical vapor deposition (MW-PACVD) reactor dedicated for high-quality born-doped diamond thin film growth. The standard BDD synthesis conditions were as follows: a microwave power of $8 \mathrm{~kW}$, a stage temperature of $850{ }^{\circ} \mathrm{C}$, a chamber pressure of $65 \mathrm{Torr}$, and a gas chemistry of $1 \%$ methane $\left(\mathrm{CH}_{4}\right)$ in a hydrogen $\left(\mathrm{H}_{2}\right)$ balance. Diborane $\left(\mathrm{B}_{2} \mathrm{H}_{6}\right)$ was added to the diamond growth process in a $\mathrm{B} / \mathrm{C}$ ratio of $37,500 \mathrm{ppm}$ to achieve sufficiently high conductivity. After the BDD synthesis, aluminum was thermally evaporated (Auto 306; Edward, Inc., West Sussex, UK) and patterned via ultraviolet (UV) photolithography (ABM-USA, Inc., San Jose, CA, USA), followed by wet chemical etching in an aluminum etchant (Type A; Transene Co., Inc., Danvers, MA, USA). With the patterned aluminum mask, the BDD film was plasma etched in an electron cyclotron resonance reactive ion etcher (RIE) using $\mathrm{SF}_{6} / \mathrm{Ar} / \mathrm{O}_{2}$ with a microwave power of $1000 \mathrm{~W}$ and a radio-frequency (RF) bias of $150 \mathrm{~W}(180 \mathrm{~V})$. Afterwards, the aluminum mask was removed using the aluminum etchant before the $\mathrm{SiO}_{2}$ was etched in a buffered oxide etchant (BOE). The $\mathrm{SiO}_{2}$ layer was slightly overetched undercutting the BDD structures to form Parylene anchors for the subsequent transfer processes.

Figure $7 \mathrm{~b}$ depicts the transfer process where the prepatterned BDD structures were transferred onto Parylene $\mathrm{C}$ films with the growth side exposed as the sensing electrodes. For this purpose, $\sim 15 \mu \mathrm{m}$ Parylene $\mathrm{C}$ was chemically vapor deposited on the patterned BDD film at 
room temperature (PDS 2010, Specialty Coating System, Inc., Indianapolis, IN, USA). Prior to Parylene deposition, the wafer was treated with a Silane A174 adhesion promoter (Sigma Aldrich, Inc., St. Louis, MO, USA). Titanium/copper was thermally evaporated and patterned to form a mask for the plasma etching of Parylene C. Parylene was etched utilizing an oxygen RF plasma with an overall power of $300 \mathrm{~W}$ and gas flow rate of $\sim 50 \mathrm{sccm}$ (PX-250; Nordson March, Inc., Concord, CA, USA). This mild plasma etch was chosen to minimize the damage to the BDD surface since oxygen plasma also etches diamond. Preparing for silicon removal, the Parylene $\mathrm{C}$ coating on the backside of the silicon wafer was removed with the oxygen plasma using the above recipe. The wafer was then placed upside down into a custom-made jig and sealed with an O-ring. The exposed silicon substrate was completely etched in $35 \% \mathrm{KOH}$ at $70{ }^{\circ} \mathrm{C}$ to release the BDD-Parylene $\mathrm{C}$ film. After removing the residual $\mathrm{SiO}_{2}$ from the nucleation side, aluminum was deposited on the growth side, followed by the deposition of another $10 \mu \mathrm{m}$ Parylene $C$ layer to encapsulate the exposed nucleation side. The BDD-Parylene film was then glued on a silicon carrier wafer using photoresist, with the growth side facing upwards. The Parylene $C$ on top of aluminum was plasma etched, followed by aluminum removal using an aluminum etchant to expose the BDD growth side as the recording electrodes. Finally, the BDD-Parylene film was released from the carrier substrate by dissolving the sacrificial photoresist in acetone and rinsed with isopropyl alcohol and deionized water. To make a fair comparison, microelectrode probes where the BDD nucleation side was exposed were fabricated using BDD films from the same MW-PACVD batch based on our previously developed method ${ }^{42}$.

\section{Material characterization}

The surface morphology of the BDD nucleation and growth sides was studied using SEM (6610 V; JEOL Inc., Peabody, MA, USA). The surface chemistry of both sides was analyzed using Raman spectroscopy (532 nm Laser; HORIBA Scientific Inc., Kyoto, Japan). The surface resistivity of the BDD film was measured using a fourpoint probe. The electrochemical impedance of the electrodes was measured using EIS (CHI604; CH Instruments, Inc., Austin, TX, USA). The electrochemical properties of the BDD electrodes were characterized using CV (CHI604; CH Instruments, Inc., Austin, TX, USA) in a three-electrode configuration. During the experiments, the DA solution was prepared by dissolving DA in a $0.1 \mathrm{M}, \mathrm{pH}=7.4$ PBS buffer solution. The effect of AA interference on DA detection was evaluated using SWV measurements (CHI604; $\mathrm{CH}$ Instruments, Inc., Austin, TX, USA). Experimental solutions were prepared by serially diluting DA in a mixture of $100 \mu \mathrm{M}$ AA and $0.1 \mathrm{M}$,
$\mathrm{pH}=7.4$ PBS. For the three-electrode setup, an $\mathrm{Ag} / \mathrm{AgCl}$ reference electrode (CHI 111; $\mathrm{CH}$ Instruments, Inc., Austin, TX, USA) was used, while a commercially available Pt wire (CHI 102; CH Instruments, Inc., Austin, TX, USA) was used as the counter electrode. Hexaammineruthenium (III) chloride $\left(\mathrm{Ru}\left(\mathrm{NH}_{3}\right)_{6} \mathrm{Cl}\right), \mathrm{KCl}, \mathrm{DA}$, and AA were purchased from Sigma Aldrich.

\section{In vitro biocompatibility assessment}

To assess the growth and attachment of neurons on the Parylene and diamond materials used in the device, E18 embryonic rat cortical neurons (\#A1084002; Thermo Fisher Scientific, Waltham, MA, USA) were cultured on the surface of planar substrates fabricated from these materials following poly-D-lysine coating according to the supplier's protocol (\#A3890401; Thermo Fisher Scientific, Waltham, MA, USA). Neurons plated on poly-D-lysinecoated Nunc Thermanox plastic coverslips (\#174950; Thermo Fisher Scientific, Waltham, MA, USA) were used as a control. Neurons were fixed with $4 \%$ paraformaldehyde in PBS at the 7-day time point and stained for beta III-tubulin (TUJ1) and caspase-3 to assess the neurite outgrowth and viability, respectively. Immunohistochemistry was performed using previously reported methods ${ }^{52}$; the primary antibodies used were a monoclonal mouse anti-beta III-tubulin antibody ("TUJ1", \#ab78078; Abcam, Cambridge, UK) at a 1:500 dilution and a rabbit cleaved caspase-3 antibody (\#9661S; Cell Signaling Technology, Inc., Danvers, MA, USA) at a 1:400 dilution. Similarly, the secondary antibodies used were Alexa Fluor goat anti-rabbit 594 (\#A-11037; Thermo Fisher Scientific, Waltham, MA, USA) at a 1:200 dilution and Alexa Fluor anti-mouse 488 (\#A-11001; Thermo Fisher Scientific, Waltham, MA, USA) at a 1:200 dilution. Sholl analysis was performed to assess the neurite outgrowth according to published protocols ${ }^{59}$, based on previous studies that demonstrate its utility as a measure of biocompatibility ${ }^{60}$. Briefly, concentric circles with defined radii of $10 \mu \mathrm{m}$ were centered on the neuronal soma and used to manually benchmark the length, branching, and intersection of the neurite outgrowth. The "maximal distance" is defined as a measurement of the maximum distance that any individual neurite extends from the soma. The "number of branching" is defined as a measurement of how many branches are formed in each particular bin. An average of $\sim 60$ neurons for each condition was randomly selected and analyzed if the neurites were clearly identifiable.

\section{In vitro electrophysiology recording}

For these experiments, rat cortical neurons were cultured on plastic coverslips, and whole-cell patch-clamp electrophysiology was performed using previously reported methods ${ }^{52}$. While the patch electrode accessed the 
neuronal membrane, a flexible BDD probe was carefully positioned adjacent to the recorded neuron in the solution using a separate micromanipulator. The patch digitizer was programmed to deliver a $5 \mathrm{~V}$ TTL pulse to an Intan RHA 2000 board $100 \mathrm{~ms}$ prior to the delivery of a $500 \mathrm{~ms}$ epoch of intracellular stimulation. At the same time, the extracellular activity was recorded via the BDD electrodes using an Intan RHA2216 amplifier chip and RHA 2000 evaluation board (Intan Technologies LLC, Los Angeles, CA, USA).

\section{In vivo electrophysiology recording}

To validate the device functionality for in vivo neural recording, the flexible diamond probe was implanted in the V1 of an adult Sprague Dawley rat (male, 550 g) using stereotaxic surgery procedures that were approved by the Institutional Animal Care and Use Committee (IACUC) at Michigan State University. During the surgery, the rat was fixed on a stereotaxic frame and anesthetized with a $2-4 \%$ isoflurane and oxygen mixture. A $3-4 \mathrm{~cm}$ incision was made in the skin overlying the skull, and a unilateral craniotomy was made on the left V1 (lateral: $3.6 \mathrm{~mm}$, anteroposterior: $6.3 \mathrm{~mm}$ relative to Bregma) using a precision surgical drill. To remove the dura mater, the bevel of a 30-gauge sterile syringe needle was pressed against a solid surface to form a small hook. The dura mater was carefully pierced and lifted using the hook and then cut using microscissors ${ }^{61}$ to expose the cortical tissue. For device implantation, the flexible probe was coated with polyethylene glycol (PEG) to temporarily stiffen the device during insertion into the cortex. The PEG-coated probe was placed in a micromanipulator mounted on the stereotaxic frame and manually lowered to the desired stereotaxic location of the brain. During the insertion, sterile saline was applied on the surface of the brain to dissolve the PEG coating from a small portion of the implant above the brain surface as the probe was advanced to the desired depth of the $\mathrm{V}^{62}$. For neural recording, the BDD electrodes were connected to a commercial 32-channel Intan recording chip, and the recorded signals were amplified and digitalized by an Intan RHD2132 system (Intan Technologies, Los Angeles, CA, USA). A ground electrode was attached to the tissue around the neck area. In some recording trials, the right eye of the rat was stimulated with LED light pulses, and the left eye was covered to avoid direct exposure to the light stimulus.

\section{Conclusion}

In this paper, we designed, fabricated, and tested a flexible BDD neural probe, where the BDD growth surface was utilized as the electrode site for neurophysiology and neurochemical sensing with potentially improved sensitivity and stability. A fabrication method was developed to transfer the BDD patterns from the solid silicon substrate onto the flexible polymer substrate and to expose the BDD growth side as the sensing surface. The general material properties and electrochemical characteristics were compared between the BDD nucleation and growth surfaces. Our results demonstrated that the electrodes made of the BDD growth side exhibited a rougher topology, a higher $s p^{3}$ content, and a larger grain size than the nucleation side. All of these results are attributed to the better sensitivity and stability of the BDD growth side electrodes for DA sensing and neural recording. In particular, for electrochemical sensing, a high-quality diamond surface with $s p^{3}$ carbon enables a wide working potential window, low background noise (due to the low double-layer capacitance), and a resistance to chemical fouling. Consequently, electrodes made of the BDD growth surface are expected to provide a wide polarizable range of chemical reactions in electrochemical sensing with improved sensitivity and reliability. On the other hand, the nanoscale surface roughness and large grain size increase the effective surface area of the electrode, therefore reducing the electrochemical impedance for electrophysiology recording with reduced impedance noise. The neural recording capability of the BDD growth side electrodes was validated both in vitro and in vivo. This flexible BDD microelectrode technology is expected to provide a unique tool for simultaneous neurophysiology and neurotransmitter sensing in neuronal circuits, which can open up numerous opportunities for fundamental and clinical research on a wide variety of brain disorders and diseases, such as Parkinson's disease.

\section{Acknowledgements}

This work was supported in part by the National Institutes of Health (NIH R21NS096637-02) and the Michigan State University_Fraunhofer USA, Inc. Center for Coatings and Diamond Technologies. E.P., C.H.T., and M.S. were partially supported by NIH/NINDS (1R01NS10745101A1) and the Departments of Biomedical Engineering and Electrical and Computer Engineering at Michigan State University. The authors also acknowledge Weiyang Yang for helping with electrochemical impedance measurements.

\section{Author details \\ 'Department of Electrical and Computer Engineering, Michigan State University, East Lansing, MI, USA. ${ }^{2}$ Fraunhofer USA Center for Coatings and Diamond Technologies, East Lansing, MI, USA. ${ }^{3}$ Department of Biomedical Engineering, Michigan State University, East Lansing, MI, USA. ${ }^{4}$ Department of Physiology, Michigan State University, East Lansing, MI, USA}

\section{Author contributions}

Unless otherwise stated, B.F. designed and fabricated the presented BDDParylene electrode probes and performed benchtop experiments to characterize the electrochemical properties of the device. C.A.R. and B.F. conducted the electrochemical experiments to evaluate the performance of the flexible BDD electrodes for DA sensing. C.H.T. and E.P. conducted the in vitro patch-clamp experiments to validate the ability to use the device for extracellular recording from cultured cortical neurons. M.S. and E.P. performed the material biocompatibility study and data analysis. Y.G. assisted in the device fabrication. R.R. and M.F.B. were responsible for the BDD film growth and material characterization and were also involved in device fabrication. Y.G. A.J.W., and W.L. conducted the in vivo animal experiments to validate the ability to use the device for extracellular recording from the primary visual 
cortex of a rat. A.J.W., M.F.B., E.P., and W.L. also supervised and advised on the concept, design, and experiments.

\section{Conflict of interest}

The authors declare that they have no conflict of interest.

Supplementary information accompanies this paper at https://doi.org/ 10.1038/s41378-020-0155-1.

Received: 19 December 2019 Revised: 6 March 2020 Accepted: 25 March 2020

Published online: 13 July 2020

\section{References}

1. Kandel, E. R. et al. Principles of Neural Science, Vol. 4 (McGraw-Hill, New York, 2000).

2. Graeff, F. G. Neuroanatomy and neurotransmitter regulation of defensive behaviors and related emotions in mammals. Braz. J. Med. Biol. Res. 27, 811-829 (1994).

3. Willis, W. D. Role of neurotransmitters in sensitization of pain responses. Ann. N. Y. Acad. Sci. 933, 142-156 (2001).

4. Brown, R. P. \& Mann, J. J. A clinical perspective on the role of neurotransmitters in mental disorders. Psychiatr. Sen. 36, 141-150 (1985).

5. Kim, J.-H. et al. Dopamine neurons derived from embryonic stem cells function in an animal model of Parkinson's disease. Nature 418, 50 (2002).

6. Mehler-Wex, C., Riederer, P. \& Gerlach, M. Dopaminergic dysbalance in distinct basal ganglia neurocircuits: implications for the pathophysiology of Parkinson's disease, schizophrenia and attention deficit hyperactivity disorder. Neurotox. Res. 10, 167-179 (2006).

7. Starr, M. S. The role of dopamine in epilepsy. Synapse 22, 159-194 (1996).

8. Jorfi, M., Skousen, J. L., Weder, C. \& Capadona, J. R. Progress towards biocompatible intracortical microelectrodes for neural interfacing applications. J. Neural Eng. 12, 011001 (2014).

9. Weltman, A., Yoo, J. \& Meng, E. Flexible, penetrating brain probes enabled by advances in polymer microfabrication. Micromachines 7, 180 (2016).

10. Subbaroyan, J., Martin, D. C. \& Kipke, D. R. A finite-element model of the mechanical effects of implantable microelectrodes in the cerebral cortex. J. Neural Eng. 2, 103 (2005).

11. Lee, J. H., Kim, H., Kim, J. H. \& Lee, S.-H. Soft implantable microelectrodes for future medicine: prosthetics, neural signal recording and neuromodulation. Lab Chip 16, 959-976 (2016).

12. Patel, P. R. et al. Insertion of linear $8.4 \mu \mathrm{m}$ diameter 16 channel carbon fiber electrode arrays for single unit recordings. J. Neural Eng. 12, 046009 (2015).

13. Guitchounts, G., Markowitz, J. E., Liberti, W. A. \& Gardner, T. J. A carbon-fiber electrode array for long-term neural recording. J. Neural Eng. 10, 046016 (2013).

14. Kozai, T. D. Y. et al. Ultrasmall implantable composite microelectrodes with bioactive surfaces for chronic neural interfaces. Nat. Mater. 11, 1065 (2012).

15. Strong, T. D., Cantor, H. C. \& Brown, R. B. A microelectrode array for real-time neurochemical and neuroelectrical recording in vitro. Sens. Actuators A: Phys. 91, 357-362 (2001).

16. Myers, R., Adell, A. \& Lankford, M. Simultaneous comparison of cerebral dialysis and push-pull perfusion in the brain of rats: a critical review. Neurosci. Biobehav. Rev. 22, 371-387 (1998).

17. Justice, J. Quantitative microdialysis of neurotransmitters. J. Neurosci. Methods 48, 263-276 (1993).

18. Kehr, J. A survey on quantitative microdialysis: theoretical models and practical implications. J. Neurosci. Methods 48, 251-261 (1993).

19. Torto, N., Majors, R. \& Laurell, T. Microdialysis sampling-challenges and new frontiers. LCGC North Am. 19, 462 (2001).

20. Heien, M. L. et al. Real-time measurement of dopamine fluctuations after cocaine in the brain of behaving rats. Proc. Natl. Acad. Sci. USA 102 10023-10028 (2005).

21. Suzuki, A. et al. Fabrication, characterization, and application of boron-doped diamond microelectrodes for in vivo dopamine detection. Anal. Chem. 79, 8608-8615 (2007).

22. Safavi, A., Maleki, N., Moradlou, O. \& Tajabadi, F. Simultaneous determination of dopamine, ascorbic acid, and uric acid using carbon ionic liquid electrode. Anal. Biochem. 359, 224-229 (2006).
23. Nimbalkar, S. et al. Ultra-capacitive carbon neural probe allows simultaneous long-term electrical stimulations and high-resolution neurotransmitter detection. Sci. Rep. 8, 6958 (2018).

24. Jacobs, C. B., Vickrey, T. L. \& Venton, B. J. Functional groups modulate the sensitivity and electron transfer kinetics of neurochemicals at carbon nanotube modified microelectrodes. Analyst 136, 3557-3565 (2011).

25. Kotov, N. A. et al. Nanomaterials for neural interfaces. Adv. Mater. 21, 3970-4004 (2009).

26. Voge, C. M. \& Stegemann, J. P. Carbon nanotubes in neural interfacing applications. J. Neural Eng. 8, 011001 (2011).

27. Bareket-KerenL. \& HaneinY. Carbon nanotube-based multi electrode arrays for neuronal interfacing: progress and prospects. Front. Neural Circuits 6, 122 (2012).

28. Vreeland, R. F. et al. Biocompatible PEDOT: Nafion composite electrode coatings for selective detection of neurotransmitters in vivo. Anal. Chem. 87, 2600-2607 (2015)

29. Zhao, H., Bian, X., Galligan, J. J. \& Swain, G. M. Electrochemical measurements of serotonin $(5-H T)$ release from the guinea pig mucosa using continuous amperometry with a boron-doped diamond microelectrode. Diam. Relat. Mater. 19, 182-185 (2010).

30. Arumugam, P. U. et al. Characterization of ultrananocrystalline diamond microsensors for in vivo dopamine detection. Appl. Phys. Lett. 102, 253107 (2013).

31. Kaivosoja, E. et al. Carbon thin films as electrode material in neural sensing. Surf. Coat. Technol. 259, 33-38 (2014).

32. Khan, S., Bazaz, S. A., Aslam, D. M. \& Chan, H. In Proc. Saudi International Electronics, Communications and Photonics Conference (SIECPC) 1-4 (IEEE, 2013).

33. Bennet, K. E. et al. A diamond-based electrode for detection of neurochemicals in the human brain. Front. Hum. Neurosci. 10, 102 (2016).

34. Soh, K. et al. Diamond-derived ultramicroelectrodes designed for electrochemical analysis and bioanalyte sensing. Diam. Relat. Mater. 17, 900-905 (2008).

35. Fujishima, $\mathrm{A}$. et al. Electroanalysis of dopamine and NADH at conductive diamond electrodes. J. Electroanal. Chem. 473, 179-185 (1999).

36. Xu, J. et al. Peer reviewed: boron-doped diamond thin-film electrodes. Anal. Chem. 69, 591A-597A (1997).

37. Dong, H., Wang, S., Galligan, J. J. \& Swain, G. M. Boron-doped diamond nano/ microelectrodes for bio-sensing and in vitro measurements. Front. Biosci. 3, 518 (2011).

38. Einaga, Y., Foord, J. S. \& Swain, G. M. Diamond electrodes: diversity and maturity. MRS Bull. 39, 525-532 (2014).

39. Wild, C. \& Wörner, E. The CVD Diamond Booklet. www.diamond-materials.com download (2004).

40. Hess, A. E., Sabens, D. M., Martin, H. B. \& Zorman, C. A. Diamond-on-polymer microelectrode arrays fabricated using a chemical release transfer process. $J$ Microelectromechanical Syst. 20, 867-875 (2011).

41. Bergonzo, P. et al. 3D shaped mechanically flexible diamond microelectrode arrays for eye implant applications: the MEDINAS project. IRBM 32, 91-94 (2011).

42. Fan, B. et al. Large-scale, all polycrystalline diamond structures transferred onto flexible Parylene-C films for neurotransmitter sensing. Lab Chip 17, 3159-3167 (2017).

43. Qi, Y. et al. Enhanced selectivity of boron doped diamond electrodes for the detection of dopamine and ascorbic acid by increasing the film thickness. Appl. Surf. Sci. 390, 882-889 (2016).

44. Bernard, M., Deneuville, A. \& Muret, P. Non-destructive determination of the boron concentration of heavily doped metallic diamond thin films from Raman spectroscopy. Diam. Relat. Mater. 13, 282-286 (2004).

45. Cobb, S. J., Ayres, Z. J. \& Macpherson, J. V. Boron doped diamond: a designer electrode material for the twenty-first century. Annu. Rev. Anal. Chem., https:// doi.org/10.1146/annurev-anchem-061417-010107 (2018).

46. Pletcher, D. A First Course in Electrode Processes (Royal Society of Chemistry, London, GB, 2009).

47. Swain, G. M. \& Ramesham, R. The electrochemical activity of boron-doped polycrystalline diamond thin film electrodes. Anal. Chem. 65, 345-351 (1993).

48. Popa, E., Notsu, H., Miwa, T., Tryk, D. \& Fujishima, A. Selective electrochemical detection of dopamine in the presence of ascorbic acid at anodized diamond thin film electrodes. Electrochem. Solid State Lett. 2, 49 (1998).

49. Trouillon, R., Einaga, Y. \& Gijs, M. A. Cathodic pretreatment improves the resistance of boron-doped diamond electrodes to dopamine fouling. Electrochem. Commun. 47, 92-95 (2014). 
50. Purcell, E. K. et al. Combining topographical and genetic cues to promote neuronal fate specification in stem cells. Biomacromolecules 13, 3427-3438 (2012).

51. Rusinek, C. A. et al. All-diamond microfiber electrodes for neurochemical analysis. J. Electrochem. Soc. 165, G3087-G3092 (2018).

52. Thompson, C. H., Khan, S. A., Khan, W. A., Li, W. \& Purcell, E. K. In Proc. 8th International IEEE/EMBS Conference on Neural Engineering (NER) 154-157 (IEEE, 2017).

53. Thompson, C., Patel, P. R., Chestek, C. A., Li, W. \& Purcell, E. K. Toward guiding principles for the design of biologically-integrated electrodes for the central nervous system. J. Neural Eng. 17, 021001 (2020)

54. Henze, D. A. et al. Intracellular features predicted by extracellular recordings in the hippocampus in vivo. J. Neurophysiol. 84, 390-400 (2000).

55. Hunt, D. L. et al. Multimodal in vivo brain electrophysiology with integrated glass microelectrodes. Nat. Biomed. Eng. 3, 741-753 (2019).

56. Vardi, R., Goldental, A., Sardi, S., Sheinin, A. \& Kanter, I. Simultaneous multipatch-clamp and extracellular-array recordings: Single neuron reflects network activity. Sci. Rep. 6, 36228 (2016).
57. Breckenridge, L. et al. Advantages of using microfabricated extracellular electrodes for in vitro neuronal recording. J. Neurosci. Res. 42, 266-276 (1995).

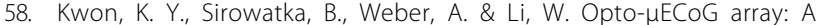
hybrid neural interface with transparent $\mu \mathrm{ECoG}$ electrode array and integrated LEDs for optogenetics. IEEE Trans. Biomed. Circuits Syst. 7, 593-600 (2013)

59. Senut, M.-C. et al. Lead exposure disrupts global DNA methylation in human embryonic stem cells and alters their neuronal differentiation. Toxicol. Sci. 139, 142-161 (2014).

60. Cesca, F. et al. Fabrication of biocompatible free-standing nanopatterned films for primary neuronal cultures. RSC Adv. 4 45696-45702 (2014).

61. Gaje, G. J. et al. Surgical implantation of chronic neural electrodes for recording single unit activity and electrocorticographic signals. J. Vis. Exp. 60, 3565 (2012).

62. Hirschberg, A. W. et al. In Proc. IEEE 30th International Conference on Micro Electro Mechanical Systems (MEMS) 129-132 (IEEE, 2017). 